\section{Poster abstracts}

\section{MEDICINE USE AND MEDICINE RELATED PROBLEMS IN PATIENTS WITH LIVER CIRRHOSIS: A SYSTEMATIC REVIEW OF QUANTITATIVE AND QUALITATIVE STUDIES}

${ }^{1}$ Ejaz Cheema, ${ }^{1}$ Abdullah Al-Hamid, ${ }^{2}$ Aliaa Al-Aryan. ${ }^{1}$ University of Birmingham; ${ }^{2}$ University of Hertfordshire

10.1136/postgradmedj-2018-fpm. 12

Introduction Liver cirrhosis is a major chronic disease that is associated with high morbidity and mortality. ${ }^{1}$ The associated co-morbidities and medical complications together with the use of complex and multiple drug therapies by patients with liver cirrhosis put them at an increased risk of developing medicine related problems (MRPs). MRPs can be defined as an event or circumstance involving drug therapy that actually or potentially interferes with desired health outcomes. ${ }^{2}$ This review aims to systematically investigate the prevalence, causes and risk factors of MRPs in cirrhotic patients and to explore factors influencing the medicine use from patients and health care providers' perspectives.

Subjects and methods Eight online databases (PubMed, Scopus, CINAHL, The British library, PsycINFO, Web of Science, EMBASE, and Google scholar) were searched up to 30th Sep, 2018 with no start date. Both quantitative studies including randomised controlled trials (RCTs), clinical trials and observational studies as well as qualitative studies were included in the review. Search terms used included 'medicine related problems', 'medicine use', 'patients' perceptions' and 'adverse drug reaction'. Data collected included the study design, baseline characteristics of study population and study outcomes. Appropriate Critical Appraisal Skills Programme (CASP) tools were used to assess both the quality of quantitative studies and qualitative studies. An in-depth descriptive analysis of the quantitative data and a thematic analysis of the qualitative data were undertaken.

Result 16 quantitative studies and 11 qualitative studies were included in the review. Of the 16 quantitative studies, nine examined the prevalence, causes, risk factors and medicine classes associated with MRPs and the remaining seven studies reported factors affecting medicine use. Mean frequency of MRPs reported in the quantitative studies ranged from 14\%23.4\%. The most frequent causes of MRPs identified in the studies included drug interactions, inappropriate dosing and use of contraindicated drugs. Major risk factors associated with MRPs included polypharmacy, severity of liver disease and length of hospital stay. Diuretics, analgesics and sedatives were suspected to be among the most common drug classes associated with MRPs. The thematic synthesis of qualitative data identified three key themes: patient related factors including lack of patients' knowledge about liver cirrhosis and its risk factors; health care related factors including lack of communication between healthcare professionals and patients; stigma and negativity including negative attitudes (misconceptions and discriminations) and negative consequences of liver cirrhosis.

Conclusions MRPs pose a significant social and financial burden on both patients and healthcare systems. Findings of this review suggest that the management of liver cirrhosis should not only be limited to the provision of safe and effective drug therapy but should also expand to improving the understanding and awareness of patients about the disease. Future research should focus on assessing the impact of patient- specific education on medicine use by patients with liver cirrhosis.

\section{REFERENCES}

1. Murray CJ, Vos T, Lozano R, et al. Disability-adjusted life years (DALYs) for 291 diseases and injuries in 21 regions, 1990-2010: a systematic analysis for the Global Burden of Disease Study 2010. Lancet 2012;380:2197-2223.

2. Strand L, Morley P, Cipolle R, Ramsey R, Lamsam G. Drug-related problems: their structure and function. DICP 1990;24:1093-1097.

\section{POMPHOLYX - A RARE SIDE EFFECT OF TRASTUZUMAB}

Abubakkar Raheel, Syed IIfan Wafa, Anupama Gore. University Hospitals of Leicester, Leicester Royal Infirmary

\subsection{6/postgradmedj-2018-fpm. 13}

Background Trastuzumab is a monoclonal antibody prescribed as part of systemic treatment of breast cancer. Trastuzumab can be prescribed on its own or as an adjuvant with chemotherapy or hormone therapy against over-expressing HER2 positive breast cancer. Patients on Trastuzumab can develop side effects of nausea, vomiting, cardiotoxicity and flu-like symptoms. ${ }^{1}$ However, we describe a rare side effect of Pompholyx (dyshidrotic eczema). This may be the first case of this presentation to be reported.

Case presentation A 50-year-old Caucasian female with a Background of $3 \mathrm{~mm}$ invasive ductal carcinoma of left breast presented with extensive vesicles and blistering on palms of hands and feet following treatment with trastuzumab [figure 1. She had wide local excision of a $3 \mathrm{~mm}$ invasive ductal carcinoma of left breast in January 2018 and sentinel lymph node biopsy which revealed a grade 2 , HER 2 positive, node negative, breast cancer. She received 6 cycles of adjuvant chemotherapy with epirubicin and cyclophosphamide followed by 15 fractions of 40 Gy adjuvant radiotherapy. Thereafter she was commenced on targeted therapy with trastuzumab. After the 2nd cycle of trastuzumab she developed multiple small vesicular eruptions predominantly on the hands and feet, which worsened by the 3 rd cycle. These were diagnosed by a dermatologist as extensive pompholyx, which was treated with topical steroid, emollient, potassium permanganate and fludroxycortide tape on the palms. Her swab cultures grew Staphylococcus aureus and betahemolytic streptococcus group-A, which was subsequently treated with intravenous flucloxacillin with a modest response.

Discussion Trastuzumab is a humanized monoclonal antibody that blocks HER2 receptors, undermining HER2 signaling in HER2 over-expressing breast cancers. The pathogenesis of pompholyx ${ }^{2}$ is not well understood thus it is difficult to establish a direct connection with trastuzumab at this stage. According to some immunohisto chemistry studies, strong presence of CD3, CD8, CD45 as well as anti-human antibodies are found in pompholyx whereas an immune mediated T-Cell lymphocyte-driven response in suppressing tumor growth in cancer has extensively been described. We plan to

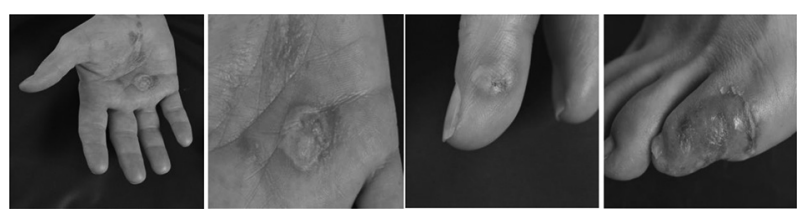

Abstract 2 Figure 1 Multiple cutaneous lesions on the palm, finger and toe. Images consented for teaching in the healthcare context. () University Hospitals of Leicester NHS Trust (Dept of Medical Illustration) 
biopsy the lesions and subsequently monitor her response to steroids with continuation of trastuzumab, since benefits of trastuzumab outweigh the risks of eczema. ${ }^{3}$

Conclusions We report the first case of trastuzumab therapy presenting with extensive infected pompholyx (dyshidrotic) eczema in a breast cancer patient. Although the patient described a history of a mild eczema five years previously, an acute flare while undergoing treatment could certainly be added to conditions at risk of flare during trastuzumab therapy until this is proven as a clear trastuzumab side effect through metaanalysis of multiple studies and a comprehensive dataset.

\section{REFERENCES}

1. Baselga J, Cortés J, Kim SB, et al, CLEOPATRA Study Group. Pertuzumab plus trastuzumab plus docetaxel for metastatic breast cancer. $N$ Engl J Med 2012;366:109-19. doi:10.1056/NEJMoa1113216

2. Ana Maria Abreu-Velez, Frank J Pinto Jr, Michael S. Howard. Dyshidrotic eczema: relevance to the immune response in situ. N Am J Med Sci 2009;1:117-120. PMCID: PMC3364640.

3. Chang HR. Trastuzumab-based neoadjuvant therapy in patients with HER2-positive breast cancer. Cancer 2010;116:2856-67. doi:10.1002/cncr.25120

\section{EVALUATION OF HO-1 INVOLVEMENT IN MELANOMA RESISTANCE TO BRAF INHIBITOR VEMURAFENIB}

Giulia Loi, AnnaLisa Furfaro, Maria Adelaide Pronzato, Gabriella Pietra, Mariapaola Nitti. Department of Experimental Medicine, Section of General Pathology, University of Genoa, Via Leon Battista Alberti, 216132 Genova (GE), Italy

\subsection{6/postgradmedj-2018-fpm.14}

Introduction The point mutation of the BRAF gene (BRAFV600E) is present in more than 50\% of melanoma.

This mutation determinate the continuous activation of MAPK pathway, which favours cell survival and proliferation. Vemurafenib/PLX4032 (PLX4032) is an inhibitor of BRAFV600 used for the therapy of these tumours, but, after an initial positive response, in many cases a relapse of the disease occurs. HO-1 is the inducible form of heme oxygenase and overexpression is involved in the growth and resistance to therapy of different types of tumours. In this study we have evaluated the involvement of HO-1 in chemoresistance and angiogenesis of BRAFV600E primary melanoma cells.

Materials and methods We used a primary melanoma cell line, derived from a metastasis from a patient, and exposed cells to PLX4032 $(1-10 \mu \mathrm{M})$. The role of HO-1 was studied by gene silencing or using the pharmacological inhibitor of HO-1 tinmesoporphyrin IX (SnMP-IX), a drug used to treat hyperbilirubinemia. Angiogenic potential was evaluated by using bovine aortic endothelial cells (BAEC) isolated in our laboratory.

Results Primary melanoma cells (MeOV-1) were exposed to 1$10 \mu \mathrm{M}$ PLX4032 for 24 hour. MTT and Trypan blue assays showed a reduction in cell viability of $45 \%$ and immunoblot and qRTPCR analyses revealed a significant upregulation of HO-1 expression. HO-1 silencing or exposure to $10 \mu \mathrm{M}$ SnMP-IX further decreased cell viability after exposure to PLX4032. Subsequently, we evaluated the angiogenetic potential of melanoma cells. Medium derived from MeOV-1 cells, exposed to 10 microlar PLX4032, was used to treat BAEC seeded on Matrigel. Tube formation, branching and density were increased in comparison to BAEC exposed to the medium from untreated MeOV-1 cells. Interestingly, when BAEC were treated with conditioned medium from MeOV-1 cells and HO-1 was silenced, $10 \mu \mathrm{M}$ PLX4032 showed a reduced ability to form tubes.
Conclusions These Results highlight an important role of HO1 in favouring melanoma resistance to target therapy and angiogenesis.

Supported by the University of Genoa, Department of Experimental Medicine.

\section{WHICH INTENSIVE CARE ANALYTICS ARE A WORTHWHILE INVESTMENT FOR DEVELOPERS? AN EARLY HEALTH TECHNOLOGY ASSESSMENT}

Lytske Bakker, Jos Aarts, Ken Redekop. Erasmus School of Health Policy and Management, Erasmus University

\subsection{6/postgradmedj-2018-fpm. 15}

Introduction In intensive care (IC), monitoring data are continuously being collected. Unfortunately, this data is often not interpretable for clinicians and therefore goes unused. While advanced analytics can process this data to support improvements in patient care, developers ideally invest in those analytics that lead to the greatest health benefits or cost reductions. We explored the potential cost-effectiveness of three IC analytics.

Subjects and methods We assessed the cost-effectiveness of three applications: the early identification of catheter-related bloodstream infection (CRBSI), improved nutrition monitoring and identification of high driving pressure (HDP) in ventilated patients. The literature was used to populate the decision trees and patient data from a Greek IC were used for the nutrition and HDP estimates. We performed univariate, multivariate, and probabilistic sensitivity analyses.

Results Early identification of CRBSI reduced mortality and length of stay, thereby increasing quality-adjusted life-years (QALYs) (6.70 vs. 6.58) and reducing costs $(€ 8183$ vs. $€ 9,869)$; probability of CRBSI and the costs per IC day were key influencers of the ICER and the probability of dominance was $90 \%$. Improved nutrition monitoring could increase nutrition adherence $(0.37 \%$ vs. $0.03 \%)$ and decrease costs by reducing length of stay $(€ 9988$ vs. $€ 10,720)$. For HDP, benefits were limited, resulting in higher costs as well as minimal and highly uncertain health benefits.

Conclusions Developers should study the potential impact of the analytics they wish to develop before investing time and money. We illustrate that analytics for early identification of CRBSI and improved nutrition monitoring are worth further investment while identifying HDP may not be. The other factors that influence success besides health and financial benefits such as the competition and data access should be assessed.

This research was part of the European AEGLE project and received funding from the European Union's Horizon 2020 Research and Innovation Programme under grant agreement No. 644906.

\section{INVESTIGATING THE EFFECT OF CATECHOLAMINES ON ISCHAEMIA-INDUCED VENTRICULAR FIBRILLATION WHEN IN THE PRESENCE OF AN INTRAVENTRICULAR BALLOON}

William Pickles, Michael Curtis. King's College London, UK

10.1136/postgradmedj-2018-fpm.16

Introduction Sudden cardiac death (SCD) is the greatest cause of cardiovascular disease-related mortality. Lethal ventricular 